CLINICAL STUDY

\title{
Serum and urinary concentrations of calprotectin as markers of insulin resistance and type 2 diabetes
}

\author{
Francisco J Ortega, Mónica Sabater, José M Moreno-Navarrete, Neus Pueyo, Patricia Botas ${ }^{1}$, Elias Delgado ${ }^{1}$,
} Wifredo Ricart, Gema Frühbeck ${ }^{2}$ and José Manuel Fernández-Real

Department of Diabetes, Endocrinology and Nutrition (UDEN), Institut d'Investigació Biomédica de Girona (IdIBGi), CIBER de la Fisiopatología de la Obesidad y la Nutrición (CIBERobn, CBO6/03/0010) and Instituto de Salud Carlos III (ISCIII), Girona, Spain, ${ }^{1}$ Hospital Central de Asturias, Oviedo, Spain and ${ }^{2}$ Clínica Universitaria de Navarra, CIBER de la Fisiopatología de la Obesidad y la Nutrición (CIBERobn, CBO6/03/0010) and Instituto de Salud Carlos III (ISCIII), Pamplona, Spain

(Correspondence should be addressed to J M Fernández-Real who is now at Section of Diabetes, Endocrinology and Nutrition, Hospital of Girona 'Dr Josep Trueta', Carretera de França s/n, 17007 Girona, Spain; Email: jmfernandezreal.girona.ics@gencat.cat)

\begin{abstract}
Objective: Increased circulating calprotectin has been reported in obese subjects but not in association with measures of insulin resistance and type 2 diabetes (T2D). The main aim of this study was to determine whether calprotectins in plasma and urine are associated with insulin resistance. Design: We performed both cross-sectional and longitudinal (diet-induced weight loss) studies. Methods: Circulating calprotectin concentrations (ELISA), other inflammatory markers, homeostasis model assessment of insulin resistance (HOMA-IR), and parameters of glucose and lipid metabolism were evaluated in 298 subjects (185 with normal (NGT) and 62 with impaired (IGT) glucose tolerance and 51 T2D subjects). Calprotectin was also evaluated in urine samples from 71 participants (50 NGT and 21 subjects with IGT). Insulin sensitivity ( $S_{I}$, Minimal Model) was determined in a subset of 156 subjects, and the effects of weight loss were investigated in an independent cohort of obese subjects $(n=19)$.

Results: Circulating calprotectin was significantly increased in IGT-T2D (independently of BMI) and positively associated with HOMA-IR, obesity measures, inflammatory markers, and parameters of glucose and lipid metabolism. Similar findings were reported for calprotectin concentrations in urine. In the subset of subjects, the association of calprotectin with $S_{I}$ was independent of BMI and age. In fact, $S_{I}$ together with C-reactive protein contributed to $27.4 \%$ of calprotectin variance after controlling for age and blood neutrophils count. Otherwise, weight loss led to decreased circulating calprotectin in parallel to fasting glucose and HOMA-IR.

Conclusion: These findings suggest that circulating and urinary concentrations of calprotectin are linked to chronic low-grade inflammation and insulin resistance beyond obesity.
\end{abstract}

European Journal of Endocrinology 167 569-578

\section{Introduction}

It is well known that chronic subclinical inflammation is intrinsic to the metabolic syndrome (the clustering of central obesity and alterations of glucose and lipid metabolism). Insulin resistance is central to the pathophysiology of these alterations, which runs together with the accumulation of fat and the presence of specific components $(1,2)$.

The innate immune system is one of the first lines of defense against invading microorganisms. In recent years, it has become evident that abnormalities in the function of the innate immune system are intrinsically linked to metabolic pathways (3). Among factors implicated in modulation of the immune response, bactericidal/permeability-increasing protein, lipopolysaccharide binding protein, complement factors, $\alpha$-defensins, and lactoferrin are proteins of the innate immune system, which are also related to metabolic parameters in humans $(4,5,6,7)$.

Calprotectin is a heterodimer composed by two calcium-binding cytoplasmic calgranulins (S100A8 and S100A9), mainly expressed in cells of the myeloid lineage (including monocytes and neutrophils), and endothelial cells in a tissue/cell-specific manner (8). The heterocomplex S100A8/A9 is secreted to the milieu in response to inflammation (8), participating in the transendothelial accumulation of monocytes at the site of inflammation (9). The molecular characterization of the S100-like domain, the translocation of phosphorylated calprotectin chains to the membrane during neutrophil activation, and its calcium-dependent association to cytoskeleton structures suggest intracellular signal transduction functions $(10,11)$. Moreover, calprotectin interacts with heparin and heparan sulfate glycosaminoglycans, the receptor for advanced 
glycation end products (RAGEs), the scavenger receptor CD36, and the toll-like receptor 4 (12).

Elevated calprotectin levels in plasma have been reported for a variety of chronic inflammatory conditions including rheumatoid arthritis, allograft rejections, inflammatory bowel disease, cancer, and lung diseases (13, 14, 15, 16). Recently, increased circulating calprotectin concentration has been found in obese subjects (17, 18). Nijhuis et al. (18) evaluated the activation state of neutrophils in nine lean and 37 morbidly obese subjects and after bariatric surgery-induced weight loss in nine obese subjects. Circulating calprotectin concentration (as a marker for neutrophil activation) was significantly increased in morbidly obese subjects and decreased after weight loss (18). Mortensen et al. (17) reported that circulating calprotectin concentration was associated with BMI in individuals without type 2 diabetes (T2D; 62 nonobese and 41 obese subjects aged between 50 and 60 years old). They found no significant association between circulating calprotectin and parameters of glucose homeostasis (17).

On the other hand, urinary and fecal concentrations of calprotectin have been reported as early markers of disease activity for conditions associated with inflammation and increased neutrophil activity (8). Elevated urinary excretion of biomarkers in T2D is related to several processes including renal damage, oxidative stress, chronic low-grade inflammation, and endothelial damage (19). Urine as a diagnostic medium allows for noninvasive detection of biomarkers, including some associated with systemic diseases such as obesity, insulin resistance, and T2D. Thereby, markers in urine that potentially offer greater sensitivity, earlier detection, or greater predictability in the development of T2D are needed.

To our knowledge, urinary calprotectins have not been evaluated in this field. Indeed, although differences for circulating calprotectin according to insulin resistance and T2D have not been reported yet, calprotectins act as ligands of the receptor for RAGEs, able to mediate detrimental effects caused by the activation of NF- $\kappa B$ via the RAGE-MAPK signaling pathway (13). As circulating and urinary calprotectins are closely associated with inflammation and may mediate detrimental effects caused by the activation of inflammatory pathways, including insulin resistance, vascular damage, and renal dysfunction, we hypothesized associations of calprotectin concentrations in both plasma and urine with obesity, insulin resistance (assessed by homeostasis model assessment of insulin resistance (HOMA-IR)), and T2D.

\section{Materials and methods}

\section{Patient recruitment}

Data and samples from 298 Caucasian men were obtained from population-based prospective studies performed in two regions of Spain between 1996 and
1999: 71 subjects were recruited from the Northwest (Asturias) (20) and 227 from Northeast (Girona) (7). Two hundred and fifty (84\%) eligible participants were selected at random from the census and, after a screening visit, they were invited to participate. The mean age of the participants was $52 \pm 12$ years. A $75 \mathrm{~g}$ oral glucose tolerance test (OGTT) according to the American Diabetes Association Criteria was performed as described previously (6). Subjects with normal glucose tolerance (NGT; $n=160$ ) had fasting plasma glucose $<7.0 \mathrm{mM}$ and 2 -h post-load plasma glucose $<7.8 \mathrm{mM}$ after a $75 \mathrm{~g}$ OGTT. Impaired glucose tolerance (IGT) was diagnosed in 62 subjects according to the American Diabetes Association Criteria (post-load glucose between 7.8 and $11.1 \mathrm{mM}$ ). Previously unknown T2D was diagnosed in 28 additional subjects (post-load glucose higher than $11.1 \mathrm{mM}$ ). Inclusion criteria were i) absence of systemic disease other than obesity and T2D and ii) absence of infection within the previous month. None of the control subjects were under medication or had evidence of metabolic disease other than obesity. Liver disease and thyroid dysfunction were specifically excluded by biochemical work-up. Insulin resistance was calculated in all subjects using the HOMA-IR value (glucose $(\mathrm{mmol} / \mathrm{l}) \times$ insulin $(\mathrm{mU} / \mathrm{l}) /$ 22.5), as previously described (21).

Twenty-five NGT and 23 T2D supplementary men were prospectively recruited from obese, diabetes, and insulin-resistant outpatient clinics on the basis of a stable metabolic control in the previous 6 months, as defined by stable $\mathrm{HbA1c}$ and fasting glucose values. Data from these patients were merged with those from the recently diagnosed IGT and T2D subjects (IGT-T2D subjects). Thereby, estimate statistical power for single comparisons of circulating calprotectin concentrations between NGT and IGT-T2D subjects increased from 80.3 to $93.8 \%$. Exclusion criteria for these patients included the following: i) clinically significant hepatic, neurological, endocrinological, or other major systemic disease, including malignancy; ii) history or current clinical evidence of hemochromatosis; iii) history of drug or alcohol abuse, defined as $>80 \mathrm{~g} /$ day in men and $>60 \mathrm{~g} /$ day in women; iv) an elevated serum creatinine concentration; v) acute major cardiovascular event in the previous 6 months; vi) acute illnesses and current evidence of acute or chronic inflammatory or infective diseases; and vii) mental illness rendering the subjects unable to understand the nature, scope, and possible consequences of the study. All subjects gave written informed consent and the protocol was approved by the Ethics Committee of the Hospital Dr Josep Trueta of Girona (Girona; Spain).

\section{Anthropometric measurements}

BMI was calculated as weight divided by height (in meters) squared. The subjects' waist was measured with a soft tape midway between the lowest rib and the 
iliac crest. The hip circumference was measured at the widest part of the gluteal region. The waist-to-hip ratio (WHR) was then calculated. Bioelectric impedance and/or air-displacement plethysmography were used to estimate body fat composition in those subjects. According to these anthropometric parameters, subjects were classified as nonobese $\left(\mathrm{BMI}<30.0 \mathrm{~kg} / \mathrm{m}^{2}\right)$ and obese subjects. Blood pressure was measured in the supine position on the right arm after a 10-min rest; a standard sphygmomanometer of appropriate cuff size was used and the first and fifth phases were recorded. Values used in the analysis are the average of three readings taken at 5-min intervals. Patients were requested to withhold alcohol and caffeine for at least $12 \mathrm{~h}$ before the different tests.

\section{Insulin sensitivity}

Insulin sensitivity $\left(S_{I}\right)$ was measured using the frequently sampled intravenous glucose tolerance test (FSIVGTT) on a different day in those subjects who agreed $(n=156,64 \%$ of the whole cohort). In brief, basal blood samples were drawn at -15 and $-5 \mathrm{~min}$, after which glucose (300 mg/kg body weight) was injected over $1 \mathrm{~min}$ starting at time 0 . At $20 \mathrm{~min}$, regular insulin $(0.03 \mathrm{U} / \mathrm{kg}$; Actrapid, Novo, Hellerup, Denmark) was injected as a bolus. Additional samples were obtained from a contralateral antecubital vein at times 1, 2, 3, 4, 5, 6, 7, 8, 10, 12, 14, 16, 19, 20, 22, 23, 24, 25, 27, 30, 40, 50, 60, 70, 80, 90, 100, 120, 140,160 , and $180 \mathrm{~min}$. Samples were rapidly collected via a three-way stopcock connected to the butterfly needle. Data from the FSIVGTT were submitted to computer programs that calculate the characteristic metabolic parameters by fitting glucose and insulin to the minimal model that describes the time course of glucose and insulin concentrations. The glucose disappearance model, by accounting for the effect of insulin and glucose on glucose disappearance, provides the parameters $S_{I}\left(10^{-4}\right)$ per minute per microunit per milliliter or the $S_{I}$ index, a measure of the effect of insulin concentrations above the basal level to enhance glucose disappearance. The estimation of model parameters was performed according to the MINMOD computer program (22).

\section{Study of the effects of weight loss}

Nineteen Caucasian obese volunteers (nine men and ten women) with NGT attending the Endocrinology Department at the University Clinic of Navarra were recruited. Patients underwent a clinical assessment including medical history, physical examination, body composition analysis, and comorbidity evaluation, as well as nutritional interviews performed by a multidisciplinary consultation team. All subjects were nonsmokers. Patients with signs of infection were excluded.
Obese patients were not receiving statins or antidiabetic medication.

Weight loss was achieved by prescription of a diet for 12 months providing a daily energy deficit of 500-1000 kcal/day as calculated from the determination of the resting energy expenditure through indirect calorimetry (Vmax29, SensorMedics Corporation, Yorba Linda, CA, USA) and multiplication by 1.4 as indicated for sedentary individuals to obtain the patient's total energy expenditure. This hypocaloric regime allows a safe and steady weight loss of $0.5-1.0 \mathrm{~kg} /$ week when followed and supplied 30, 54, and $16 \%$ of energy requirements in the form of fat, carbohydrates, and protein respectively. Body weight was measured with a digital scale to the nearest $0.1 \mathrm{~kg}$ and height was measured to the nearest $0.1 \mathrm{~cm}$ with a Holtain stadiometer (Holtain Ltd., Crymych, UK). All subjects gave written informed consent after the purpose of the study was explained to them. The institutional review board of the University Clinic of Navarra (Navarra; Spain) approved the protocol, so we certify that all applicable institutional regulations concerning the ethical use of information and samples from human volunteers were followed during this research. Complete clinical trial registration is deposited into ClinicalTrials.gov (accession number: NCT01572090).

\section{Analytical determinations}

The serum glucose levels were measured in duplicate by the glucose oxidase method with a Beckman Glucose Analyzer 2 (Beckman Coulter Inc., Brea, CA, USA). The coefficient of variation (CV) was $1.9 \%$. The serum insulin levels were measured in duplicate by monoclonal IRMA (Medgenix Diagnostics, Fleunes, Belgium). The lowest limit of detection was $4.0 \mathrm{mU} / \mathrm{l}$. The intra-assay CVs was $5.2 \%$ at a concentration of $10 \mathrm{mU} / \mathrm{l}$ and $3.4 \%$ at $130 \mathrm{mU} / \mathrm{l}$. The interassay $\mathrm{CV}$ were 6.9 and $4.5 \%$ at 14 and $89 \mathrm{mU} / \mathrm{l}$ respectively.

Total serum cholesterol was measured through the reaction of cholesterol esterase/oxidase/peroxidase, using a BM/Hitachi 747. HDL-cholesterol was quantified after precipitation with polyethylene glycol at room temperature. Total serum triglycerides were measured through the reaction of glycerol-phosphate-oxidase and peroxidase. Whole blood hemoglobin levels (EDTA sample, Coulter Electronics, Hialeah, FL, USA) were determined by routine laboratory tests. Plasma samples were collected using EDTA as anticoagulant and centrifuged for $15 \mathrm{~min}$ at $1000 \mathrm{~g}$ within $30 \mathrm{~min}$ of collection. Aliquots of $500 \mu \mathrm{l}$ were immediately done and stored at $-80{ }^{\circ} \mathrm{C}$ for further measures. Urine samples were stored at $-80{ }^{\circ} \mathrm{C}$ upon receipt for longlasting storage. Frozen plasma and urine aliquots were thawed on ice before measurements. Samples were not submitted to freeze-thaw cycles. 
Table 1 Clinical characteristics of subjects in the cross-sectional study. Data are mean \pm s.D.

\begin{tabular}{|c|c|c|c|c|}
\hline & $\begin{array}{l}\text { Nonobese } \\
(\mathrm{BMI}<30)\end{array}$ & $\begin{array}{c}\text { Obese } \\
(\mathrm{BMI} \geq 30)\end{array}$ & $P$ & $\begin{array}{c}P \\
(\mathrm{M}-\mathrm{W})\end{array}$ \\
\hline$n$ & 201 & 97 & & \\
\hline Age (years) & $51 \pm 12$ & $52 \pm 11$ & 0.481 & 0.028 \\
\hline $\mathrm{BMI}\left(\mathrm{kg} / \mathrm{m}^{2}\right)$ & $25.9 \pm 2.4$ & $36.4 \pm 7$ & $<0.0001$ & $<0.0001$ \\
\hline Fat mass $(\%)$ & $26.7 \pm 4.3$ & $39.6 \pm 7.6$ & $<0.0001$ & $<0.0001$ \\
\hline Waist $(\mathrm{cm})$ & $88.8 \pm 7.6$ & $114.8 \pm 16.9$ & $<0.0001$ & $<0.0001$ \\
\hline Hip (cm) & $97.3 \pm 5.9$ & $113.7 \pm 18.2$ & $<0.0001$ & $<0.0001$ \\
\hline WHR & $0.91 \pm 0.1$ & $1 \pm 0.1$ & $<0.0001$ & $<0.0001$ \\
\hline $\mathrm{SBP}(\mathrm{mmHg})$ & $126.2 \pm 16.8$ & $138.8 \pm 19.1$ & $<0.0001$ & $<0.0001$ \\
\hline $\mathrm{DBP}(\mathrm{mmHg})$ & $78.2 \pm 9.8$ & $85.8 \pm 12$ & $<0.0001$ & $<0.0001$ \\
\hline Serum glucose (mg/dl) & $99.2 \pm 17.4$ & $104.8 \pm 30.4$ & 0.092 & 0.008 \\
\hline Insulin (mIU/ml) & $8.2 \pm 4.9$ & $18 \pm 20.2$ & $<0.0001$ & $<0.0001$ \\
\hline HbA1c (\%) & $4.9 \pm 0.7$ & $5.4 \pm 0.9$ & $<0.0001$ & $<0.0001$ \\
\hline Insulin sensitivity ${ }^{\mathrm{a}}$ & $3 \pm 1.8$ & $1.6 \pm 1.3$ & $<0.0001$ & $<0.0001$ \\
\hline HOMA-IR & $2 \pm 1.3$ & $3.8 \pm 2.3$ & $<0.0001$ & $<0.0001$ \\
\hline Total cholesterol (mg/dl) & $204.7 \pm 38.5$ & $209.2 \pm 37.7$ & 0.345 & 0.044 \\
\hline HDL-cholesterol (mg/dl) & $53.2 \pm 13.4$ & $47 \pm 11.3$ & $<0.0001$ & 0.007 \\
\hline LDL-cholesterol (mg/dl) & $130.7 \pm 34.5$ & $132.8 \pm 36.8$ & 0.631 & 0.091 \\
\hline Fasting triglycerides $(\mathrm{mg} / \mathrm{dl})$ & $111.3 \pm 109.9$ & $138.5 \pm 66.3$ & 0.025 & 0.045 \\
\hline Blood neutrophil count $(\mathrm{U} / \mu \mathrm{l})$ & $3816 \pm 1754$ & $4453 \pm 2132$ & 0.013 & 0.001 \\
\hline Blood lymphocyte count $(U / \mu l)$ & $1704 \pm 725$ & $1970 \pm 613$ & 0.005 & 0.089 \\
\hline Blood monocyte count (U/ $/ \mu \mathrm{l})$ & $453 \pm 277$ & $522 \pm 175$ & 0.043 & 0.016 \\
\hline C-reactive protein (mg/l) & $0.31 \pm 0.4$ & $0.5 \pm 0.5$ & $<0.0001$ & 0.003 \\
\hline Circulating calprotectin (ng/ml) & $102.8 \pm 71.7$ & $131.4 \pm 63.7$ & $<0.0001$ & 0.028 \\
\hline Calprotectin $(\mathrm{ng} / \mathrm{ml})$ in urine ${ }^{\mathrm{b}}$ & $5.2 \pm 4.9$ & $19.2 \pm 26.4$ & 0.001 & 0.034 \\
\hline
\end{tabular}

WHR, waist-to-hip ratio; SBP, systolic blood pressure; DBP, diastolic blood pressure; HOMA-IR, homeostasis model assessment of insulin resistance value. *, Student's $t$-test.

a Insulin sensitivity was measured in 156 subjects (101 subjects with normal and 55 subjects with impaired glucose tolerance or T2D) using the FSIVGTT. Significant data are shown in bold.

${ }^{\mathrm{b}}$ Calprotectin in urine was measured in 71 subjects (24 subjects with normoweight and 47 subjects with obesity).

Significant data, for Student's $t$-test or nonparametric comparisons (Mann-Whitney $U$ test), are shown in bold.

A solid-phase ELISA based on the sandwich principle (Hycult Biotech; Uden, The Netherlands) was used for the in vitro quantitative determination of human calprotectin in plasma and urine samples. High sensitivity C-reactive protein (CRP) was also analyzed by ELISA kits (BLK Diagnostics; Barcelona, Spain). Intra- and inter-assay $\mathrm{CV}$ for all these determinations were under $10 \%$.

\section{Statistical analyses}

Descriptive results of continuous variables are expressed as mean (s.D.). Before statistical analysis, normal distribution and homogeneity of the variances were evaluated using Levene's test and then variables were given a base $\log _{10}$ transformation, if necessary. These parameters ( $S_{I}$ and circulating calprotectin) were analyzed on a $\log$ scale and tested for significance on that scale. The anti-log-transformed values of the means (geometric mean) are reported in the following tables. The relationship between variables was tested using Pearson's test and stepwise multiple linear regression analysis. We used ANOVA and the unpaired $t$-tests for comparisons of quantitative variables. The statistical significance of differences reported between groups was also evaluated by nonparametric test (Mann-Whitney $U$ test), in addition to t-test. General linear models were performed to calculate circulating calprotectin concentrations after adjusting for several variables. The statistical analyses were performed using the program SPSS (version 13.0 SPSS Inc., Chicago, IL, USA).

\section{Results}

\section{Circulating calprotectin concentration}

The metabolic and clinical characteristics of the subjects included in the cross-sectional study are shown in Table 1. Circulating calprotectin concentrations were skewed to the left in the population studied. $\log _{10^{-}}$ transformed calprotectin followed a normal curve and was then used in all the analyses performed. Circulating calprotectin concentration increased according to the insulin resistance/adiposity status of the subjects (Fig. 1a). Circulating calprotectin was increased in plasma from obese compared with nonobese subjects $(102.8 \pm 71.7$ vs $131.4 \pm 63.7 \mathrm{ng} / \mathrm{ml}, \quad P<0.0001$; Table 1) and these differences remained significant even when subjects with T2D were excluded from the analysis $(99.3 \pm 70.1$ vs $124.1 \pm 53.9 \mathrm{ng} / \mathrm{ml}$, $P=0.002$ ). Indeed, calprotectin concentration was positively associated with anthropometric, biochemical, 
A

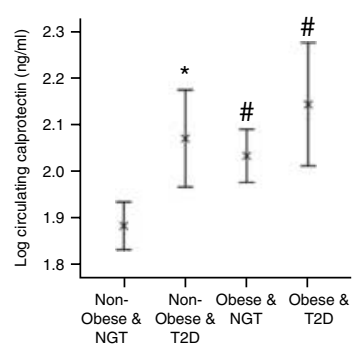

D

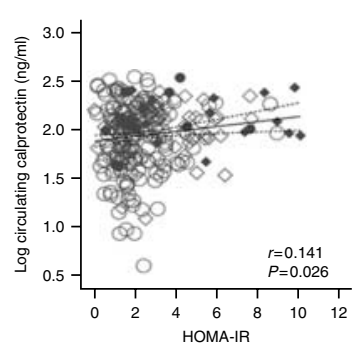

B

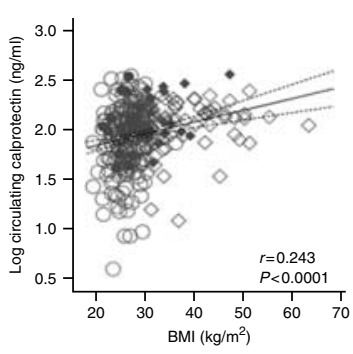

E

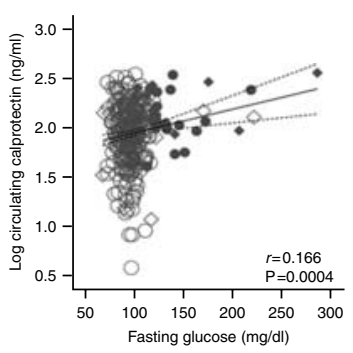

C

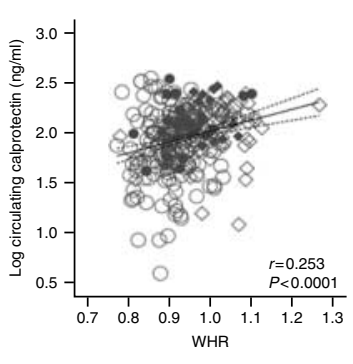

$\mathrm{F}$

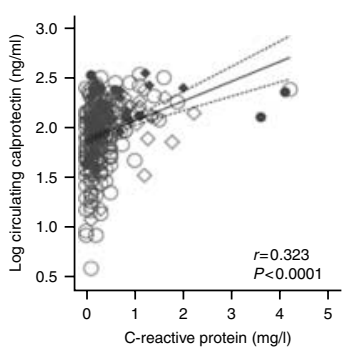

Figure 1 Error bars: mean and 95\% confidence interval for the mean of $\log _{10}$-transformed circulating calprotectin concentrations $(\mathrm{ng} / \mathrm{ml})$ in (a) nonobese $\left(\mathrm{BMI}<30 \mathrm{~kg} / \mathrm{m}^{2}\right)$ and obese $\left(\mathrm{BMI} \geq 30 \mathrm{~kg} / \mathrm{m}^{2}\right)$ subjects with normal glucose tolerance (NGT) or type 2 diabetes (T2D). ${ }^{\star} P<0.05$ and ${ }^{\#} P<0.0001$ for comparisons between metabolically compromised groups and the control group (nonobese and NGT men). Scatter plots: linear relationships between $\log _{10}$-transformed circulating calprotectin and the BMI (b), the waist-to-hip ratio (c), HOMA-IR (d), fasting glucose (e), and C-reactive protein (f). Values for nonobese and NGT individuals are represented as open circles, nonobese subjects with T2D are closed circles, obese and NGT are represented as open diamonds, and obese and T2D patients as closed diamonds. and inflammatory parameters and negatively with $S_{I}$ and HDL-cholesterol (Table 2). Of note, calprotectins in plasma correlated $(r=0.23, P=0.003, n=170)$ with markers of leukocyte activity, such as the alarm secretory leukocyte protease inhibitor (23).

When stratifying by quartiles of calprotectin concentrations (cut points at 59.3, 99.0, and $150.7 \mathrm{ng} / \mathrm{ml}$ ), BMI (Fig. 1b), percent fat mass, WHR (Fig. 1c), systolic and diastolic blood pressure, and fasting triglycerides were significantly increased across calprotectin quartiles. HOMA-IR (Fig. 1d), fasting glucose (Fig. 1e), glucose at $120 \mathrm{~min}$ of the OGTT, and inflammatory parameters such as blood neutrophils count and CRP (Fig. 1f) also increased according to circulating calprotectin concentration (Table 3).

Interestingly, $S_{I}$ contributed independently to $5.1 \%$ $(P=0.006)$ of circulating calprotectin after controlling for the effects of age and BMI (Table 4) in the subpopulation of 156 men where $S_{I}$ values (Minimal Model) were available. $S_{I}(P=0.022)$ and CRP $(P<0.0001)$ accounted together for $27.4 \%$ $(P<0.0001)$ of calprotectin concentration variance in plasma after controlling for age and blood neutrophils count (Table 4). Otherwise, fasting glucose $(P<0.0001)$, blood neutrophils $(P=0.0004)$, and CRP $(P<0.0001)$ contributed independently to $27.5 \%$ $(P<0.0001)$ of circulating calprotectin variance after controlling for the effects of BMI and age in a multiple linear regression model for the whole cohort (Table 4). In subjects with obesity, fasting glucose $(P<0.0001)$ and CRP $(P=0.003)$ explained together $27.3 \%$ of circulating calprotectin variance in the same multiple linear regression model, while only blood neutrophils $(P<0.0001)$ and CRP $(P<0.0001)$ did $(28.5 \%)$ in nonobese men (both $P<0.0001$, Table 4 ).

\section{Calprotectin concentration in urine}

In a subpopulation of 71 men, calprotectin concentration was also increased in urine from obese compared with nonobese subjects $(19.2 \pm 26.4$ vs 5.2 $\pm 7.9 \mathrm{ng} / \mathrm{ml}, P=0.001)$. When subjects with T2D

Table 2 Correlation between $\log _{10}$-transformed calprotectin concentrations and study variables in the cross-sectional study.

\begin{tabular}{|c|c|c|c|c|}
\hline \multirow[b]{3}{*}{ Subjects (all men) } & \multirow{2}{*}{\multicolumn{2}{|c|}{$\begin{array}{c}\begin{array}{c}\text { Circulating } \\
\text { calprotectin }\end{array} \\
298\end{array}$}} & \multirow{2}{*}{\multicolumn{2}{|c|}{$\begin{array}{c}\begin{array}{c}\text { Calprotectin } \\
\text { in urine }\end{array} \\
71\end{array}$}} \\
\hline & & & & \\
\hline & $r$ & $P$ & $r$ & $P$ \\
\hline ge (years) & 0.050 & 0.393 & -0.022 & 0.856 \\
\hline BMI $\left(\mathrm{kg} / \mathrm{m}^{2}\right)$ & 0.243 & $<0.0001$ & 0.221 & 0.066 \\
\hline Fat mass (\%) & 0.244 & $<0.0001$ & 0.203 & 0.090 \\
\hline Waist (cm) & 0.272 & $<0.0001$ & 0.228 & 0.060 \\
\hline Hip (cm) & 0.210 & $<0.0001$ & 0.148 & 0.229 \\
\hline WHR & 0.253 & $<0.0001$ & 0.301 & 0.013 \\
\hline SBP $(m$ & 0.270 & $<0.0001$ & 0.003 & 0.981 \\
\hline $\mathrm{DBP}(\mathrm{mmHg})$ & 0.177 & 0.002 & 0.250 & 0.038 \\
\hline Fasting glucose (mg/dl) & 0.166 & 0.004 & 0.404 & $<0.0001$ \\
\hline Insulin sensitivity ${ }^{a}$ & -0.254 & 0.002 & NA & NA \\
\hline HOMA-IR & 0.141 & 0.026 & 0.382 & 0.002 \\
\hline Total cholesterol (mg/dl) & -0.067 & 0.247 & -0.255 & 0.032 \\
\hline HDL-cholesterol (mg/dl) & -0.159 & 0.006 & -0.220 & 0.065 \\
\hline LDL-cholesterol (mg/dl) & -0.082 & 0.165 & -0.309 & 0.010 \\
\hline Fasting triglycerides $(\mathrm{mg} / \mathrm{dl})$ & 0.291 & $<0.0001$ & 0.319 & 0.007 \\
\hline Blood neutrophil count $(\mathrm{U} / \mu \mathrm{l})$ & 0.316 & $<0.0001$ & -0.041 & 0.835 \\
\hline 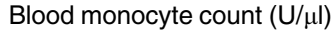 & 0.167 & 0.008 & -0.049 & 0.800 \\
\hline C-reactive protein (mg/l) & 0.323 & $<0.0001$ & 0.294 & 0.013 \\
\hline Calprotectin in urine $(\mathrm{ng} / \mathrm{ml})$ & 0.291 & 0.015 & & \\
\hline
\end{tabular}

WHR, waist-to-hip ratio; SBP, systolic blood pressure; DBP, diastolic blood pressure; NA, not available; HOMA-IR.

ansulin sensitivity was measured in 156 subjects (101 subjects with normal and 55 subjects with impaired glucose tolerance or T2D) using the FSIVGTT. Significant data are shown in bold. 


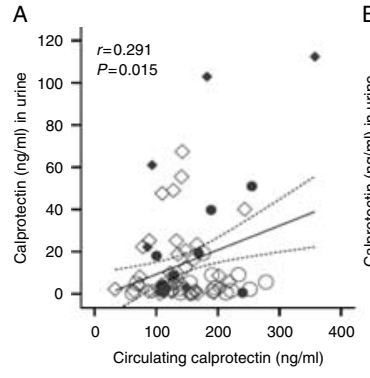

D

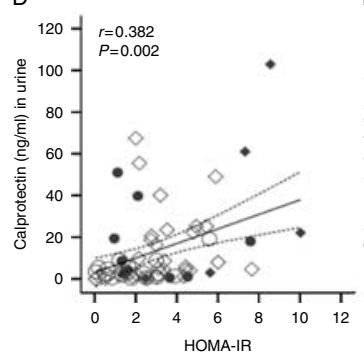

B

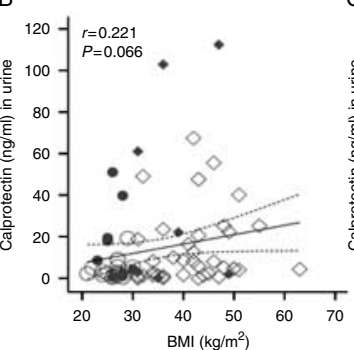

E

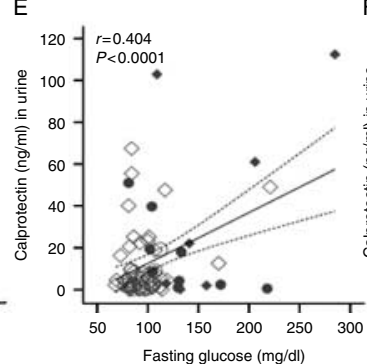

C

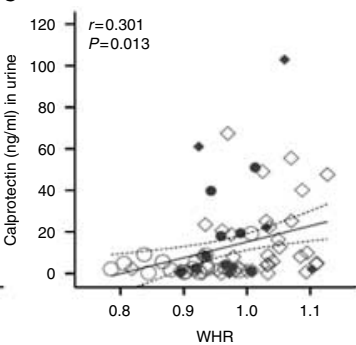

$\mathrm{F}$

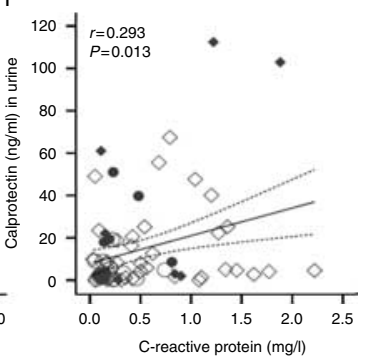

Figure 2 Linear relationships between calprotectin concentrations in urine and plasma (a) and between calprotectin in urine and BMI (b; nonsignificant), the waist-to-hip ratio (c), HOMA-IR (d), fasting glucose (e), and C-reactive protein (f). Values for nonobese and NGT individuals are represented as open circles, nonobese subjects with T2D are closed circles, obese and NGT are represented as open diamonds, and obese and T2D patients as closed diamonds. were excluded from the analysis, these differences remained significant $(15.2 \pm 18.1$ vs $5.7 \pm 6.4 \mathrm{ng} / \mathrm{ml}$, $P=0.004)$. Of note, values of urinary concentrations of calprotectin significantly correlated (Table 2) with circulating calprotectin (Fig. 2a). Indeed, calprotectin in urine was associated with BMI (Fig. 2b), WHR (Fig. 2c), diastolic blood pressure, fasting triglycerides, parameters of $S_{I}$ such as HOMA-IR (Fig. 2d) and fasting glucose (Fig. 2e), and with CRP (Fig. 2f).

\section{Weight loss study}

Characteristics of the subjects included in this longitudinal study are shown in Table 5. No significant differences were reported for circulating calprotectin concentrations between men and women neither prenor post-weight loss. No significant associations between circulating calprotectins and age were shown in men or women in this independent cohort of obese

Table 3 Clinical characteristics of subjects according to circulating calprotectin concentration. Results are presented as mean \pm s.D. for Gaussian variables and median (interquartile range) for non-Gaussian variables.

\begin{tabular}{|c|c|c|c|c|c|c|}
\hline Quartiles ( $n$ ) & $\mathbf{Q}_{1}(75)$ & $\mathbf{Q}_{2}(74)$ & $\mathbf{Q}_{3}(74)$ & $\mathbf{Q}_{\mathbf{4}}(75)$ & $\boldsymbol{P}(\mathrm{ANOVA})$ & $\boldsymbol{P}^{\star}$ \\
\hline Circulating calprotectin (ng/ml) & $39.2(25.3-48.5)$ & $80.6(68.2-89.3)$ & $123.8(110.1-132.3)$ & 198.5 (165.9-243.9) & $<0.0001$ & $<0.0001$ \\
\hline Calprotectin in urine $(\mathrm{ng} / \mathrm{ml})$ & $2.23(2.00-3.42)$ & $4.86(1.26-22.3)$ & $4.64(2.01-18.39)$ & $5.49(0.93-19.32)$ & 0.768 & 0.355 \\
\hline Age (years) & $51 \pm 10$ & $49 \pm 10.4$ & $53 \pm 13$ & $53 \pm 12$ & 0.194 & 0.908 \\
\hline BMI $\left(\mathrm{kg} / \mathrm{m}^{2}\right)$ & $26.4 \pm 3.8$ & $29.3 \pm 5.9$ & $30.9 \pm 8.7$ & $30.7 \pm 6.2$ & $<0.0001$ & $<0.0001$ \\
\hline Fat mass $(\%)$ & $27.4 \pm 5.5$ & $30.3 \pm 7.7$ & $33.1 \pm 10.1$ & $32.6 \pm 7.4$ & $<0.0001$ & $<0.0001$ \\
\hline Waist (cm) & $88.4 \pm 9.1$ & $96.3 \pm 15.6$ & $101.7 \pm 19.8$ & $100.9 \pm 15.6$ & $<0.0001$ & $<0.0001$ \\
\hline WHR & $0.91 \pm 0.07$ & $0.93 \pm 0.07$ & $0.96 \pm 0.07$ & $0.96 \pm 0.08$ & $<0.0001$ & $<0.0001$ \\
\hline $\mathrm{SBP}(\mathrm{mmHg})$ & $120.8 \pm 13.2$ & $128.9 \pm 16.2$ & $137 \pm 20.8$ & $134.2 \pm 19.1$ & $<0.0001$ & $<0.0001$ \\
\hline $\mathrm{DBP}(\mathrm{mmHg})$ & $76.2 \pm 9$ & $82.3 \pm 12.5$ & $81.4 \pm 11.3$ & $82.8 \pm 10.6$ & 0.001 & $<0.0001$ \\
\hline Fasting glucose (mg/dl) & $96.7 \pm 12.9$ & $99.6 \pm 18.7$ & $103.4 \pm 23.9$ & $103.6 \pm 30.1$ & 0.174 & 0.016 \\
\hline 120'-post glucose (mg/dl) & $120.8 \pm 38.6$ & $142.3 \pm 51.7$ & $145.3 \pm 58.4$ & $139.9 \pm 56.6$ & 0.028 & 0.003 \\
\hline Insulin $(\mathrm{mlU} / \mathrm{ml})$ & $8.8 \pm 5.01$ & $13.12 \pm 9.96$ & $11.01 \pm 7.59$ & $13.5 \pm 22.4$ & 0.137 & 0.003 \\
\hline Insulin sensitivity ${ }^{a}$ & $3.2 \pm 2.22$ & $2.19 \pm 1.66$ & $2.74 \pm 1.44$ & $2.01 \pm 1.29$ & 0.020 & 0.015 \\
\hline HOMA-IR & $2.08 \pm 1.25$ & $3.08 \pm 2.24$ & $2.64 \pm 1.86$ & $2.72 \pm 2.02$ & 0.024 & 0.001 \\
\hline HDL-cholesterol (mg/dl) & $55.7 \pm 13.8$ & $48.4 \pm 9.8$ & $50.1 \pm 12.3$ & $50 \pm 14.7$ & 0.004 & $<0.0001$ \\
\hline LDL-cholesterol (mg/dl) & $135.4 \pm 33$ & $128.2 \pm 38.1$ & $133 \pm 32.8$ & $129 \pm 37.2$ & 0.569 & 0.164 \\
\hline Fasting triglycerides (mg/dl) & $84.5 \pm 41.4$ & $118.5 \pm 65.6$ & $116.1 \pm 72.4$ & $123.6 \pm 61.5$ & $<0.0001$ & $<0.0001$ \\
\hline Blood neutrophil count $(\mathrm{U} / \mu \mathrm{l})$ & $3232 \pm 1037$ & $3965 \pm 2216$ & $4225 \pm 1451$ & $4906 \pm 2345$ & $<0.0001$ & $<0.0001$ \\
\hline Blood monocyte count $(\mathrm{U} / \mu \mathrm{l})$ & $408 \pm 150$ & $493 \pm 407$ & $481 \pm 149$ & $536 \pm 178$ & 0.028 & 0.007 \\
\hline C-reactive protein (mg/l) & $0.10(0.10-0.30)$ & $0.20(0.10-0.39)$ & $0.30(0.10-0.50)$ & $0.40(0.20-0.80)$ & $<0.0001$ & $<0.0001$ \\
\hline
\end{tabular}

WHR, waist-to-hip ratio; SBP, systolic blood pressure; DBP, diastolic blood pressure. *, Student's $t$-test

${ }^{a}$ Insulin sensitivity was measured in 156 subjects (101 subjects with normal and 55 subjects with impaired glucose tolerance or T2D) using the FSIVGTT. Student's $t$-test was performed for comparisons between first quartile $\left(Q_{1}\right)$ and merged values from second, third, and fourth quartile $\left(>Q_{1}\right)$ according to circulating calprotectin concentrations. Significant data are shown in bold. 
Table 4 Multiple linear regression analyses with circulating calprotectin as dependent variable for all subjects in the cross-sectional study.

\begin{tabular}{|c|c|c|c|c|c|c|}
\hline & \multicolumn{2}{|c|}{$\begin{array}{l}\text { All subjects } \\
\quad(n=298)\end{array}$} & \multicolumn{2}{|c|}{$\begin{array}{l}\text { Nonobese subjects } \\
\qquad(n=201)\end{array}$} & \multicolumn{2}{|c|}{$\begin{array}{l}\text { Obese subjects } \\
\qquad(n=97)\end{array}$} \\
\hline & $\beta$ & $P$ & $\beta$ & $P$ & $\beta$ & $P$ \\
\hline \multicolumn{7}{|l|}{ Model I } \\
\hline Age & -0.027 & 0.622 & -0.038 & 0.572 & -0.117 & 0.308 \\
\hline BMI $\left(\mathrm{kg} / \mathrm{m}^{2}\right)$ & -0.010 & 0.873 & 0.033 & 0.621 & -0.080 & 0.540 \\
\hline Fasting glucose (mg/dl) & 0.226 & $<0.0001$ & 0.095 & 0.153 & 0.419 & $<0.0001$ \\
\hline Blood neutrophil count $(U / \mu \mathrm{l})$ & 0.208 & 0.0004 & 0.307 & $<0.0001$ & 0.025 & 0.821 \\
\hline C-reactive protein (mg/l) & 0.367 & $<0.0001$ & 0.385 & $<0.0001$ & 0.342 & 0.003 \\
\hline Adjusted $R^{2}$ & 27.4 & $<0.0001$ & 28.5 & $<0.0001$ & 27.3 & $<0.0001$ \\
\hline \multicolumn{7}{|l|}{ Model II $(n=156)$} \\
\hline Insulin sensitivity ${ }^{a}$ & -0.179 & 0.022 & & & & \\
\hline Age & -0.129 & 0.098 & & & & \\
\hline Blood neutrophil count $(\mathrm{U} / \mu \mathrm{l})$ & 0.128 & 0.108 & & & & \\
\hline C-reactive protein $(\mathrm{mg} / \mathrm{l})$ & 0.354 & $<0.0001$ & & & & \\
\hline Adjusted $R^{2}$ & 27.4 & $<0.0001$ & & & & \\
\hline \multicolumn{7}{|l|}{ Model III $(n=156)$} \\
\hline Insulin sensitivity ${ }^{a}$ & -0.232 & 0.006 & & & & \\
\hline Age & -0.062 & 0.463 & & & & \\
\hline BMI $\left(\mathrm{kg} / \mathrm{m}^{2}\right)$ & 0.147 & 0.135 & & & & \\
\hline Adjusted $R^{2}$ & 5.1 & $<0.05$ & & & & \\
\hline
\end{tabular}

$\beta$ is the standardized regression coefficient, which allows evaluating the relative significance of each independent variable in multiple linear regression analyses. Adjusted $R^{2}$ expresses the percentage of the variance explained by the independent variables in the different models (i.e. 0.50 is $50 \%$ ).

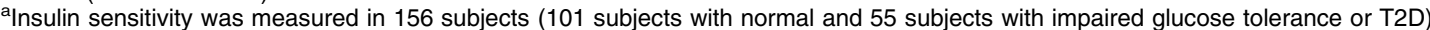
using the FSIVGTT. Significant data are shown in bold.

subjects with NGT. However, for the whole cohort, the associations of circulating calprotectin concentrations with fasting triglycerides $(r=0.494, P=0.037)$, insulin resistance $(r=-0.571, P=0.007)$, and serum glucose $(r=0.674, P=0.002)$ were replicated (Table 5). Weight loss led to significantly decreased circulating calprotectin $(-34.3 \%, P=0.011)$ (Table 5). The decrease in fasting glucose $(r=0.605, P=0.022)$ and insulin resistance levels (HOMA-IR; $r=0.583, P=0.047$ ) was parallel to that of calprotectins concentration in plasma, despite no significant changes in leucocyte count (Table 5).

\section{Discussion}

Calgranulin monomers (calgranulin A and B) show a strong preference to form a calcium-dependent heterodimer called calprotectin and is regarded as the functional relevant form at circulating levels (24). This complex has been suggested as a biomarker for inflammation and of utility in the monitoring of disease activity (25). Indeed, calprotectin, as a component of the innate immune system associated with leukocyte adhesion, chemotaxis, and phagocytosis, appears to promote recruitment and infiltration of macrophages and polymorphonuclear cells into the inflammatory lesions (26). Accordingly, calprotectins in plasma correlated $(r=0.23, P=0.003, n=170$; Fig. 3$)$ with markers of leukocyte activity such as, the alarm secretory leukocyte protease inhibitor (23).

Given that adipose tissue dysfunction is believed to act as a source of inflammatory-related complications in individuals with central obesity, who are prone to obesity-related comorbidities due to chronic inflammation $(27,28)$, we hypothesized that increased circulating calprotectin and increased concentrations

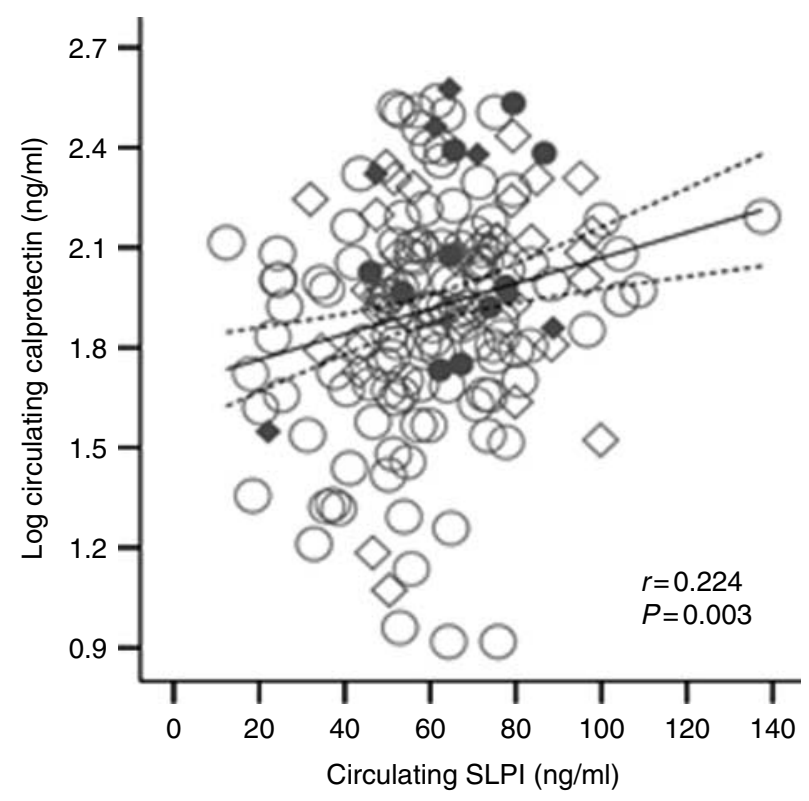

Figure 3 Correlation between calprotectin and the alarm secretory leukocyte protease inhibitor (SLPI) concentrations in plasma. Values for nonobese and NGT individuals are represented as open circles, nonobese subjects with T2D are closed circles, obese and NGT are represented as open diamonds, and obese and T2D patients as closed diamonds. 
Table 5 Subjects' characteristics in the diet-induced weight loss study. Results are presented as mean \pm s.D.

\begin{tabular}{|c|c|c|c|c|}
\hline & Baseline & $\begin{array}{l}\text { Post-weight } \\
\text { loss }\end{array}$ & $\begin{array}{l}\text { Percentage } \\
\text { of variation }\end{array}$ & $\boldsymbol{P}^{\star}$ \\
\hline Number of participants $(n)$ & & 19 (M:9; F: & & \\
\hline Age (years) & & $43 \pm 14$ & & \\
\hline Weight $(\mathrm{kg})$ & $109.8 \pm 30.3$ & $91.0 \pm 16.5$ & -17.2 & $<0.0001$ \\
\hline $\mathrm{BMI}\left(\mathrm{kg} / \mathrm{m}^{2}\right)$ & $38.3 \pm 9.2$ & $31.9 \pm 5.8$ & -16.6 & $<0.0001$ \\
\hline Fat mass $(\mathrm{kg})$ & $44.6 \pm 7.7$ & $36.9 \pm 9.5$ & -17.4 & $<0.0001$ \\
\hline Waist $(\mathrm{cm})$ & $114.7 \pm 19.4$ & $102.6 \pm 13.9$ & -10.6 & $<0.0001$ \\
\hline WHR & $0.95 \pm 0.08$ & $0.94 \pm 0.08$ & -1.6 & 0.318 \\
\hline $\mathrm{SBP}(\mathrm{mmHg})$ & $125.9 \pm 15.9$ & $122.1 \pm 15.7$ & -3.0 & 0.373 \\
\hline $\mathrm{DBP}(\mathrm{mmHg})$ & $79.3 \pm 9.9$ & $76.1 \pm 7.4$ & -4.0 & 0.186 \\
\hline Fasting glucose (mg/dl) & $98.4 \pm 11.6$ & $92.2 \pm 7.9$ & -6.3 & 0.057 \\
\hline HOMA-IR & $4.6 \pm 3.9$ & $2.7 \pm 1.3$ & -41.3 & 0.050 \\
\hline Total cholesterol (mg/dl) & $213.1 \pm 31.8$ & $182.7 \pm 29.3$ & -14.3 & 0.005 \\
\hline LDL-cholesterol (mg/dl) & $137.9+33.1$ & $112.9+25.0$ & -18.1 & 0.012 \\
\hline HDL-cholesterol (mg/dl) & $54.0 \pm 12.8$ & $54.2 \pm 10.9$ & -0.4 & 0.930 \\
\hline Fasting triglycerides (mg/dl) & $106.4 \pm 41.0$ & $77.9 \pm 29.7$ & -26.7 & 0.005 \\
\hline Blood leukocyte count $(\mathrm{mU} / \mu \mathrm{l})$ & $7.1 \pm 1.0$ & $7.4 \pm 1.3$ & +4.7 & 0.140 \\
\hline Calprotectin $(\mathrm{ng} / \mathrm{ml})$ & $305.1 \pm 283.4$ & $200.4 \pm 159.3$ & -34.3 & 0.011 \\
\hline Calprotectin in men $(\mathrm{ng} / \mathrm{ml})$ & $359.1+276.3$ & $228.3+171.2$ & -36.4 & 0.059 \\
\hline Calprotectin in women $(\mathrm{ng} / \mathrm{ml})$ & $256.5 \pm 295.3$ & $175.2 \pm 152.4$ & -31.7 & 0.119 \\
\hline
\end{tabular}

M, male; F, female; WHR, waist-to-hip ratio; SBP, systolic blood pressure; DBP, diastolic blood pressure; HOMA-IR, homeostasis model assessment of insulin resistance value. Significant data are shown in bold. *, Student's $t$-test.

in urine would be present in subjects with obesity and/or insulin resistance. Moreover, as calprotectin is able to mediate detrimental effects caused by the activation of NF- $\mathrm{KB}$, inducing pro-inflammatory gene expression (13), the increased concentrations of calprotectins in plasma and urine may also be associated with T2D and diabetic complications such as hypertension and renal failure.

In this study, the most remarkable findings were: i) the chronic low-grade inflammatory status of obesity and T2D was characterized by increased calprotectin concentrations in plasma and urine; ii) circulating calprotectin was associated with insulin resistance even after controlling for BMI and inflammatory markers, especially among obese subjects, according to multiple regression analyses; and iii) diet-induced weight loss led to significantly decreased circulating levels of calprotectins in parallel to insulin resistance, whereas leukocyte number did not change. As one goal of the T2D definition is to facilitate the clinical identification of insulin-resistant individuals, biomarkers of insulin resistance are increasingly being explored. According to current results, increased calprotectin concentration in plasma and urine may be markers of increased insulin resistance and the development of T2D, as well as an obesityassociated chronic low-grade inflammatory trait. Otherwise, proinflammatory cytokines are suggested as important factors in the development of diabetes-related renal injury (29), among other comorbidities. Then, urinary calprotectins may be a new inflammatory factor related to the development of T2D and its complications.

The positive relationships between calprotectins and inflammatory parameters, such as blood neutrophils count and CRP, suggest that immune system activation modulates circulating calprotectin concentrations. Therefore, even though circulating calprotectin seems to be mainly expressed and released by circulating polymorphonuclear cells (18), endothelial cells and macrophages in adipose tissue may be an active source responsible for the increase in circulating calprotectins in obese individuals.

In summary, our findings suggest a close interplay between chronic low-grade inflammation, insulin resistance, and calprotectin concentrations in plasma and urine. Nowadays, new biomarkers of insulin resistance, such as soluble CD14 (30) and LBP (31), among others $(4,5,6,7)$, are increasingly being explored. The findings reported here are compatible with the notion that obesity-related chronic low-grade inflammation leads to increased calprotectin concentrations in both plasma and urine. However, current data also suggest that calprotectin may be a biomarker of decreased $S_{I}$, beyond a common trait of obesity and inflammation. Further investigations will be required to evaluate the utility and specificity of calprotectin as a biomarker of insulin resistance and T2D.

\section{Declaration of interest}

The authors have nothing to disclose. All authors of this manuscript have directly participated in the execution and analysis of the study. All authors are aware of and agree to the content of the manuscript, and all authors have approved the final version submitted and their being listed as an author on the manuscript. The authors have declared that no competing interests exist.

\section{Funding}

This work was supported by research grants from the Ministerio de Educación y Ciencia (SAF2005-02073) and Generalitat de Catalunya (2005SGR00947 and 2005SGR00467). The funders had no role in study design, data collection and analysis, decision to publish, or preparation of the manuscript. 


\section{Author contribution statement}

All authors of this manuscript have directly participated in the execution and analysis of the study. F J Ortega drafted the manuscript designed the study, participated in the analysis of biochemical variables, and performed the statistical analysis. M Sabater, J M Moreno-Navarrete, and N Pueyo analyzed biochemical variables. P Botas and E Delgado obtained samples from the Spanish population from the Northwest (Asturias) and were in charge of analytical studies in this population. G Frühbeck realized the diet-induced weight loss study. W Ricart and G Frühbeck participated in the conception and the coordination of this study. J M Fernández-Real carried out the coordination of the study and helped with the manuscript.

\section{Acknowledgements}

The authors greatly appreciate the technical assistance of Gerard Pardo, Ester Guerra, and Oscar Rovira (Unit of Diabetes, Endocrinology and Nutrition. Institut d'Investigació Biomèdica de Girona, Hospital Universitari de Girona Dr Josep Trueta). The work of all the members of the Multidisciplinary Obesity Team of the Clínica Universitaria de Navarra is gratefully acknowledged.

\section{References}

1 Hotamisligil GS. Inflammation and metabolic disorders. Nature 2006444 860-867. (doi:10.1038/nature05485)

2 Shoelson SE, Lee J \& Goldfine AB. Inflammation and insulin resistance. Journal of Clinical Investigation 2006116 1793-1801. (doi:10.1172/JCI29069)

3 Fernandez-Real JM \& Pickup JC. Innate immunity, insulin resistance and type 2 diabetes. Trends in Endocrinology and Metabolism 200819 10-16. (doi:10.1016/j.tem.2007.10.004)

4 Gubern C, Lopez-Bermejo A, Biarnes J, Vendrell J, Ricart W \& Fernandez-Real JM. Natural antibiotics and insulin sensitivity: the role of bactericidal/permeability-increasing protein. Diabetes 200655 216-224. (doi:10.2337/diabetes.55.01.06.db05-1108)

5 Lopez-Bermejo A, Chico-Julia B, Castro A, Recasens M, Esteve E, Biarnes J, Casamitjana R, Ricart W \& Fernandez-Real JM. Alpha defensins 1,2, and 3: potential roles in dyslipidemia and vascular dysfunction in humans. Arteriosclerosis, Thrombosis, and Vascular Biology 200727 1166-1171. (doi:10.1161/ATVBAHA.106. 138594)

6 Moreno-Navarrete JM, Martinez-Barricarte R, Catalan V, Sabater M, Gomez-Ambrosi J, Ortega FJ, Ricart W, Bluher M, Fruhbeck G, Rodriguez de Cordoba S \& Fernandez-Real JM. Complement factor $\mathrm{H}$ is expressed in adipose tissue in association with insulin resistance. Diabetes 201059 200-209. (doi:10.2337/ db09-0700)

7 Moreno-Navarrete JM, Ortega FJ, Bassols J, Castro A, Ricart W \& Fernandez-Real JM. Association of circulating lactoferrin concentration and 2 nonsynonymous LTF gene polymorphisms with dyslipidemia in men depends on glucose-tolerance status. Clinical Chemistry 200854 301-309. (doi:10.1373/clinchem.2007. 095943)

8 Striz I \& Trebichavsky I. Calprotectin - a pleiotropic molecule in acute and chronic inflammation. Physiological Research 200453 245-253.

9 Manitz MP, Horst B, Seeliger S, Strey A, Skryabin BV, Gunzer M, Frings W, Schonlau F, Roth J, Sorg C \& Nacken W. Loss of S100A9 (MRP14) results in reduced interleukin-8-induced CD11b surface expression, a polarized microfilament system, and diminished responsiveness to chemoattractants in vitro. Molecular and Cellular Biology 200323 1034-1043. (doi:10.1128/MCB.23.3. 1034-1043.2003)
10 Guignard F, Mauel J \& Markert M. Phosphorylation of myeloidrelated proteins MRP-14 and MRP-8 during human neutrophil activation. European Journal of Biochemistry 1996241 265-271. (doi:10.1111/j.1432-1033.1996.0265t.x)

11 Kligman D \& Hilt DC. The $\mathrm{S} 100$ protein family. Trends in Biochemical Sciences 198813 437-443. (doi:10.1016/09680004(88)90218-6)

12 Leclerc E, Fritz G, Vetter SW \& Heizmann CW. Binding of S100 proteins to RAGE: an update. Biochimica et Biophysica Acta 2009 1793 993-1007. (doi:10.1016/j.bbamcr.2008.11.016)

13 Ehlermann P, Eggers K, Bierhaus A, Most P, Weichenhan D, Greten J, Nawroth PP, Katus HA \& Remppis A. Increased proinflammatory endothelial response to S100A8/A9 after preactivation through advanced glycation end products. Cardiovascular Diabetology 20065 6. (doi:10.1186/1475-2840-5-6)

14 Foell D \& Roth J. Proinflammatory S100 proteins in arthritis and autoimmune disease. Arthritis and Rheumatism $2004 \mathbf{5 0}$ 3762-3771. (doi:10.1002/art.20631)

15 Leach ST, Mitchell HM, Geczy CL, Sherman PM \& Day AS. S100 calgranulin proteins S100A8, S100A9 and S100A12 are expressed in the inflamed gastric mucosa of Helicobacter pyloriinfected children. Canadian Journal of Gastroenterology 200822 461-464.

16 Leach ST, Yang Z, Messina I, Song C, Geczy CL, Cunningham AM \& Day AS. Serum and mucosal $\mathrm{S} 100$ proteins, calprotectin (S100A8/S100A9) and S100A12, are elevated at diagnosis in children with inflammatory bowel disease. Scandinavian Journal of Gasteroenterology $2007 \mathbf{4 2}$ 1321-1331. (doi:10.1080/003655 20701416709)

17 Mortensen $\mathrm{OH}$, Nielsen AR, Erikstrup C, Plomgaard P, Fischer CP, Krogh-Madsen R, Lindegaard B, Petersen AM, Taudorf S \& Pedersen BK. Calprotectin - a novel marker of obesity. PLoS ONE 20094 e7419. (doi:10.1371/journal.pone.0007419)

18 Nijhuis J, Rensen SS, Slaats Y, van Dielen FM, Buurman WA \& Greve JW. Neutrophil activation in morbid obesity, chronic activation of acute inflammation. Obesity $2009172014-2018$. (doi:10.1038/oby.2009.113)

19 Matheson A, Willcox MD, Flanagan J \& Walsh BJ. Urinary biomarkers involved in type 2 diabetes: a review. Diabetes/ Metabolism Research and Reviews 201026 150-171. (doi:10.1002/ dmrr. 1068)

20 Valdes S, Botas P, Delgado E, Alvarez F \& Cadorniga FD. Population-based incidence of type 2 diabetes in northern Spain: the Asturias Study. Diabetes Care 200730 2258-2263. (doi:10.2337/dc06-2461)

21 Bonora E, Targher G, Alberiche M, Bonadonna RC, Saggiani F, Zenere MB, Monauni T \& Muggeo M. Homeostasis model assessment closely mirrors the glucose clamp technique in the assessment of insulin sensitivity: studies in subjects with various degrees of glucose tolerance and insulin sensitivity. Diabetes Care 200023 57-63. (doi:10.2337/diacare.23.1.57)

22 Bergman RN, Prager R, Volund A \& Olefsky JM. Equivalence of the insulin sensitivity index in man derived by the minimal model method and the euglycemic glucose clamp. Journal of Clinical Investigation 1987 79 790-800. (doi:10.1172/JCI112886)

23 Lopez-Bermejo A, Ortega FJ, Castro A, Ricart W \& Fernandez-Real JM. The alarm secretory leukocyte protease inhibitor increases with progressive metabolic dysfunction. Clinica Chimica Acta 2011412 1122-1126. (doi:10.1016/j.cca.2011. 02.037)

24 Vogl T, Leukert N, Barczyk K, Strupat K \& Roth J. Biophysical characterization of S100A8 and S100A9 in the absence and presence of bivalent cations. Biochimica et Biophysica Acta 2006 1763 1298-1306. (doi:10.1016/j.bbamcr.2006.08.028)

25 Foell D, Hernandez-Rodriguez J, Sanchez M, Vogl T, Cid MC \& Roth J. Early recruitment of phagocytes contributes to the vascular inflammation of giant cell arteritis. Journal of Pathology $2004 \mathbf{2 0 4}$ 311-316. (doi:10.1002/path.1660)

26 Halayko AJ \& Ghavami S. S100A8/A9: a mediator of severe asthma pathogenesis and morbidity? Canadian Journal of Physiology and Pharmacology 200987 743-755. (doi:10.1139/Y09-054) 
27 Gregor MF \& Hotamisligil GS. Inflammatory mechanisms in obesity. Annual Review of Immunology 201129 415-445. (doi:10.1146/ annurev-immunol-031210-101322)

28 Wellen KE \& Hotamisligil GS. Obesity-induced inflammatory changes in adipose tissue. Journal of Clinical Investigation 2003112 1785-1788. (doi:10.1172/JCI20514)

29 Navarro JF \& Mora C. Perspectives and usefulness of inflammatory biomarkers within the context of chronic renal disease. Nefrologia 200727 (Suppl 6) 8-14.

30 Fernandez-Real JM, Lopez-Bermejo A, Castro A, Broch M, Penarroja G, Vendrell J, Vazquez G \& Ricart W. Opposite relationship between circulating soluble CD14 concentration and endothelial function in diabetic and nondiabetic subjects. Thrombosis and Haemostasis 200594 615-619. (doi:10.1160/ TH05-03-0615)

31 Sun L, Yu Z, Ye X, Zou S, Li H, Yu D, Wu H, Chen Y, Dore J, Clement K, Hu FB \& Lin X. A marker of endotoxemia is associated with obesity and related metabolic disorders in apparently healthy Chinese. Diabetes Care 201033 1925-1932. (doi:10.2337/dc10-0340)

Received 2 May 2012

Revised version received 10 July 2012

Accepted 20 July 2012 